Rev. salud pública. 10 sup (1): 97-108, 2008

\title{
Anotaciones sobre la Equidad en los Resultados en Salud
}

\author{
Notes on equity in health outcomes
}

Óscar Fresneda-Bautista

Centro de Investigaciones para el Desarrollo (CID), Universidad Nacional de Colombia. Universidad Externado de Colombia. ofresneda@yahoo.com

Recibido 8 Enero 2008/Enviado para Modificación 2 Mayo 2008/Aceptado 26 Octubre 2008

\section{RESUMEN}

El artículo ubica el análisis de los resultados en salud dentro del conjunto de elementos que comprende la evaluación de la equidad en salud y sugiere algunas orientaciones para ese análisis. Toma como punto de referencia el modelo de análisis para el examen de la equidad en salud que ha adoptado el Observatorio de Equidad en Calidad de Vida y Salud de Bogotá, en el cual participa el autor. El texto aborda las siguientes preguntas: ¿Cuál es el papel que juega la evaluación de resultados en el contexto de la equidad en salud? ¿Cómo evaluar las inequidades en los resultados de salud? ¿Qué evaluar en torno a los resultados en salud? En la parte final señala cómo el tema de la equidad en salud no se agota en el examen de las desigualdades injustas entre individuos o grupos.

Palabras Clave: Desigualdades de la salud, inequidad social, evaluación en salud (Fuente: DeCS, BIREME)

\section{ABSTRACT}

The article locates analysing health outcomes within a set of elements covering evaluating equity in health and suggests some orientations for such analysis. The model of analysis for examining equity in health which has been adopted by the «Observatory for Bogotá's Equity in Quality of Life and Health» has been taken as a point of reference. The text approaches the following questions: What is the role played by evaluating health outcomes within the context of equity in health? How can inequities in health outcomes be evaluated? What should be evaluated regarding health outcomes? The final part deals with how the topic of equity in health does not become exhausted in examining unjust inequalities between individuals or groups.

Key Words: Inequality in health, social inequity, health evaluation (source: MeSH. NLM). 
n este artículo hago algunas cortas referencias sobre tres temas re-
lacionados con el papel de la evaluación de la equidad en los resulta-
dos en salud, afrontando las siguientes preguntas: 1. ¿Cuál es la pertinencia y significado de esta evaluación en la perspectiva de dar cuenta de las inequidades sociales expresadas en la salud?

¿Cuál es el papel que juega la evaluación de resultados en el contexto de la equidad en salud?

2. ¿Cómo evaluar las inequidades en los resultados de salud?

3. ¿Qué evaluar en torno a los resultados en salud? ¿Cuáles son los te mas a considerar en la evaluación de los resultados en salud?

En la parte final hago una breve ilustración tendiente a señalar cómo el tema de la equidad en salud no se agota en el examen de las desigualdades injustas entre individuos o grupos.

Incorporo discusiones y preocupaciones que desde el Grupo de Protección Social del CID de la Universidad Nacional hemos tenido en los últimos dos años. Y recojo igualmente lo que en nuestra experiencia conjunta con la Secretaría Distrital de Salud de Bogotá hemos avanzado en el diseño y puesta en operación del Observatorio de Equidad en Calidad de Vida y Salud de Bogotá (SDS-CID) (1). Nuestros debates y avances no son conclusivos y lo que presento sólo compromete al autor.

¿Cuál es el papel que juega la evaluación de resultados en la perspectiva de la equidad en salud?

La Figura 1 ilustra los elementos e interrelaciones del modelo de análisis que ha adoptado el Observatorio de Equidad en Calidad de Vida y Salud de Bogotá para el examen de la equidad en salud y que sirve de guía para identificar los temas a que deben tomarse en cuenta para las evaluaciones que se propone llevar a cabo. El modelo que allí se esboza retoma, con algunas modificaciones, el propuesto por la Diderichsen y Hallqvist $(2,3)$ y recoge aportes de los desarrollos metodológicos en que se basa la Alianza Global para el Monitoreo de la Equidad, GEGA, para hacer el seguimiento de la equidad en salud en los centros donde opera (4). La Figura 2 detalla algunos de los contenidos de los espacios de evaluación a ser abordados.

De la observación de estos esquemas se destaca, en primer lugar, la multiplicidad de asuntos que se requiere tomar en cuenta para la evaluación de la equidad en salud. Se pone de presente la complejidad que representa esta 
evaluación, al llamar a encontrar las interdependencias que hay entre ellos. La diversidad de casillas de las figuras muestra el carácter multidimensional de la evaluación y la pluralidad de temas que es abarcada al tratar de llevarla a cabo. De partida se está postulando que la equidad en salud es un concepto multidimensional para cuyo abordaje se debe tomar en cuenta una amplia gama de aspectos de la vida social relacionados entre sí (5). Su análisis no se agota en la consideración de la equidad en el acceso a servicios de salud, ni tampoco en la de las desigualdades en la situación de salud, como con frecuencia se piensa. Culminarlo implica sobrepasar la descripción, para encontrar los factores causales que explican las múltiples expresiones de las inequidades y guían las posibles acciones para superarlas.

Figura 1. Interrelaciones entre los elementos del modelo de análisis sobre las inequidades en salud

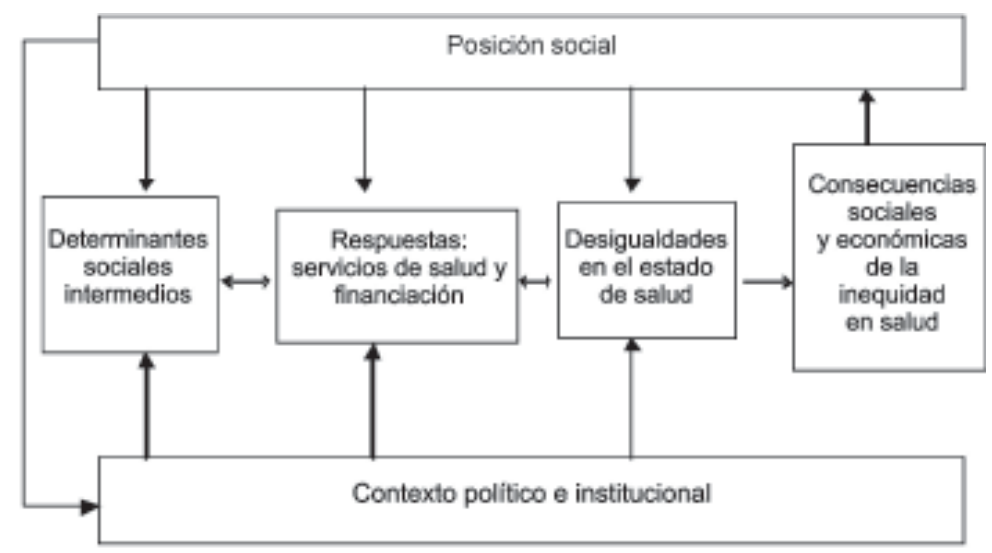

Dentro de los componentes del modelo de análisis, los resultados en salud ocupan el papel prioritario. Constituyen lo que debe ser explicado en primera instancia, como efecto de múltiples determinaciones sociales, y son ellos lo que posee un valor por sí mismo y dota de valor a otros de los elementos, que juegan un papel de determinantes y factores causales, o que son consecuencias derivadas de ese factor central. Se busca, ante todo, que las inequidades en salud sean suprimidas y que eso contribuya a una mejor salud y calidad de vida de quienes están siendo discriminados.

El otorgar este puesto a los resultados en salud no deja de lado la importancia que tiene para la evaluación la consideración de las capacidades para tener una vida adecuada. Porque la salud puede entenderse, al decir de A. 
Sen, como "un componente fundamental de las posibilidades humanas" y porque si bien la distinción entre logros en salud y posibilidades de tener buena salud “es importante en algunos casos... en la mayoria de las situaciones el logro de la salud tiende a ser una buena guía de las posiblidades subyacentes, dado que tendemos a darle prioridad a la buena salud cuando realmente tenemos la oportunidad de elegir”.(5)

Pero la evaluación la equidad en salud no se restringe al examen de las potencialidades y los resultados. Requiere igualmente la consideración de los procesos que en las Figuras 1 y 2 están expresados en las “respuestas” y en el "contexto institucional”. La equidad en los procesos es complemento indispensable de la equidad en los resultados, ya que para ser lograda no puede violar la imparcialidad en las formas como se obtiene. Margaret Whitehead señala que la equidad en salud es la situación donde todo individuo tenga oportunidades imparciales ("fair opportunity”) para lograr su plena potencialidad. Y esa condición implica que es preciso acogerse a procedimientos imparciales $(1,6)$.

Figura 2. Modelo para el análisis y seguimiento de la equidad en salud Determinantes sociales estructurales



Si las mujeres, por ejemplo, tienen ciertas ventajas naturales para tener una vida más prologada, la igualdad en la duración de la vida no puede alcanzarse a costa de discriminar su acceso a los servicios de salud para dar preferencia a los hombres (5). En este caso, en aras de la equidad en los resultados, se violaría la imparcialidad en los procesos. 
La casilla de "respuestas" de la figura incluye, como parte de los procesos, el espacio de análisis de la distribución de los servicios sociales de salud. Postular que la evaluación de la equidad se debe centrar en la forma como se prestan los servicios de salud bajo el supuesto de que de ellos depende en forma decisiva el estado de salud de las personas es desconocer que su papel es instrumental y que la salud no se explica únicamente por ese factor. Los logros y fracasos en el campo de la salud dependen de otros elementos que van desde las predisposiciones genéticas, los ingresos individuales, los hábitos alimentarios y los estilos de vida, hasta el entorno epidemiológico y las condiciones de trabajo (5).

Las casillas de la izquierda de las figuras expresan algunos de esos determinantes en términos de exposición diferencial a la enfermedad y de factores que inciden desigualmente en las capacidades de las personas para lograr una plena salud. Se distinguen tres tipos de causas asignables al contexto social que apuntan a develar circunstancias que pueden ser afectadas a través de acciones de naturaleza diferente. En primer lugar, las desigualdades en “oportunidades” básicas para la salud, tendientes a destacar factores que afectan en forma permanente las posibilidades de alcanzar un pleno desarrollo de salud, como la nutrición infantil o el desarrollo físico y afectivo. Señalan factores que afectan las personas especialmente durante la infancia y la juventud y que crean desventajas permanentes y difícilmente contrarrestables. La desnutrición en la etapa de lactancia materna o en los primeros años de vida marca, por ejemplo, una predisposición que se expresa en la salud de la persona a lo largo de toda la vida.

El segundo tipo de determinante social intermedio tomado en cuenta, es la exposición diferencial. Señala la forma como pueden influir las desigualdades sociales en el estado de salud de forma que quienes se encuentran en peor situación pueden verse más expuestos a los peligros y factores de riesgo para la salud, que aumentan sus probabilidades de enfermedad y muerte prematura. Las exposiciones diferenciales pueden explicar el exceso de riesgo de mala salud asociado a las posiciones sociales más bajas (7).

El tercer tipo de determinante social intermedio, la vulnerabilidad diferencial, se refiere a condiciones sociales que dificultan la respuesta ante la enfermedad o riesgos contra la salud. Las posibilidades de respuesta para obtener una buena salud afectan los resultados en función de cuestiones como la posesión de activos, la composición familiar, los lazos de solidaridad con la comunidad próxima. Aquí se incluyen factores de desventaja que afectan las potencialidades de la salud. 
En la perspectiva de la movilización social y de la trasformación de la sociedad hacia una mayor equidad, el análisis de las inequidades debe estar orientado no solo a la denuncia social sobre los factores estructurales que explican esa situación, sino también a señalar áreas de intervención donde puedan tomarse medidas correctivas. Eso implica develar los mecanismos y situaciones concretas a través de los cuales se concretan las formas de discriminación.

¿Cómo evaluar las inequidades en los resultados de salud?

Dentro de las medidas sobre el estado de salud se encuentran los índices que integran la mortalidad y la morbilidad y analizan su distribución entre individuos. Una metodología para construir indicadores de este tipo es propuesta por Gakidou, Murray y Frenk (8) en un artículo del Boletín de la Organización Mundial de la Salud. La propuesta acoge la definición de salud poblacional que integra información sobre mortalidad y morbilidad y que ha guiado la construcción de medidas globales del estado de salud.

Ya que estas medidas agregadas son indiferentes a la distribución, no dan cuenta directamente de la desigualdad y pueden ofrecer señales equivocas para el tratamiento de personas con desventaja, así por ejemplo, la minimización de los AVAD (años de vida ajustados en función de la discapacidad), una medida de esta familia, actúa en forma "perversa" dado que, como afirma Sen, una persona discapacitada o con una enfermedad crónica, y por lo tanto con una desventaja general, si se adopta como criterio minimizar el AVAD también recibiría menos atención médica para otras enfermedades y con esto tiene se añadiría más desventaja relativa a alguien que ya está en desventaja (5). La propuesta de Gakidou, Murray y Frenk está orientada a precisar procedimientos y fuentes de información para llegar a medidas de desigualdad entre individuos a partir del concepto de salud poblacional. Los autores dan indicaciones sugestivas para la construcción empírica de esta variable, para llegar a un índice agregado de resultados en salud. La propuesta se basa en el postulado de que "las desigualdades en salud significa fundamentalmente comparar la distribución del estado de salud entre los individuos de una población y comparar las distribuciones de distintas poblaciones.” Con este enfoque y calculado la distribución de la salud poblacional pueden obtenerse indicadores de desigualdad como los utilizados para medir las desigualdades económicas, tipo coeficiente de Gini o las desviaciones individuales respecto de la media. La propuesta aún no se ha aplicado. La elaboración de índices de este tipo requiere, sin embargo, de información de que no se dispone en países como Colombia ni en la mayor parte de países latinoamericanos. Señala una perspectiva para trabajos futuros. 
Medidas de este tipo podrían dar cuenta de la magnitud general de la desigualdad en salud, lo que permitiría analizar temas como el de sus interrelaciones y dependencias con variables socioeconómicas de asignación de gasto a salud, de crecimiento económico y de desigualdad social. Pero el enfoque de la desigualdad entre individuos lleva a no tomar en cuenta otros factores más decisivos en la explicación de esas desigualdades.

La manera como informa sobre la desigualdad no dice mucho sobre los factores sociales de que depende y sobre sus determinantes estructurales sociales, ni permite un juicio sobre las desigualdades como injustas. Gakidou, Murray y Frenk, en otro artículo señalan que es útil medir las desigualdades en salud entre grupos, pero que las desigualdades en salud deberían definirse desde el punto de vista de las desigualdades inter-individuales (10). Una propuesta para incorporar estos temas al análisis es formulada por Paula Braveman y Sofía Gruskin (9). Para las autoras la equidad en salud puede ser aprendida constatando la ausencia de desigualdades sistemáticas en salud y en sus determinantes entre grupos con diferente nivel de ventajas o desventajas sociales en cuanto a riqueza, poder o prestigio. En forma operativa, la equidad de salud con esta metodología está definida como la ausencia de diferencias en salud entre los grupos menos y más desfavorecidos.

Esta definición se basa en presupuestos de causalidad social para identificar y medir las inequidades. En tal perspectiva las autoras afirman que "de acuerdo con la definición propuesta de equidad, una disparidad en salud es inequitativa si está sistemáticamente asociada con situaciones de desventaja social”. La asociación estadística permite asumir que, dentro de la compleja cadena de fenómenos que expliquen los fenómenos de salud, existe un nexo causal significativo entre las situaciones de desigualdad y las de desventaja social (9).

La identificación de las situaciones de desigualdad que puedan calificarse como inequitativas requiere, bajo este enfoque, postular una causalidad, así sea de forma hipotética: esta desigualdad es injusta porque está asociada y se explica por una diferencia social. Tal postulado, al mismo tiempo, lleva a concluir que no son simples diferencias naturales, sino expresiones de prácticas sociales discriminatorias e injustas.

Acogiendo esta forma de evaluación, la casilla superior de la figura que representa el modelo de evaluación de la equidad en salud del Observatorio señala el papel de determinante principal de la inequidad en términos de estructuras sociales. Al asignarle esa posición se postula que las desigualdades 
sociales están en la base de desigualdades en los factores determinantes intermedios y en las diferencias en los servicios de salud, que configuran un conjunto de prácticas discriminatorias, las cuales, a su vez, originan las inequidades en el estado de salud.

Las inequidades en salud son, en última instancia, un resultado de las condiciones estructurales de desigualdad social, en lo económico, político y social. El factor que representa el contexto social y particularmente la forma como las personas se encuentran ubicadas en determinadas posiciones sociales juega un papel decisivo en tanto afecta directamente las diferencias en la salud y los factores que las condicionan: determinantes sociales intermedios y “respuestas".

En todos los elementos del modelo de análisis el contexto social está presente a través de categorías de análisis que expresan las "posiciones sociales" o de categorías provisionales que están asociadas con las desigualdades de salud y sus determinantes. Es el componente de enlace que articula los análisis y que, en lo posible, está incluido en las medidas o indicadores seleccionados.

Sobre esta forma de medir las desigualdades es preciso hacer algunas acotaciones. En primera instancia, la asociación estadística no es un criterio definitivo de causación. Enfrentamos el conocido problema de las correlaciones espurias: una asociación empírica no expresa necesariamente vínculos causales. En segundo lugar, en un terreno práctico, las medidas que podemos construir, en el estado de nuestro conocimiento y con las limitaciones impuestas por la información disponible, puede haber una parte de las desigualdades en salud que tengan baja correlación o no se expliquen directamente por las variables o categorías de desventaja social que estén disponibles y sean escogidas para establecer la asociación. Y, en tercer lugar, la adopción del grupo más aventajado como patrón de referencia para identificar las inequidades, no presupone que sea el modelo para todas las circunstancias relacionadas con la salud y sus determinantes. Si, por su estilo de vida particular, tiene preferencias en relación con sus viviendas y con el consumo alimentario, que puedan caber dentro de lo que algunos economistas han calificado como "gustos caros", o están más propensos a ciertas enfermedades, esto no significa que toda la sociedad deba evaluar sus logros en relación con ellos. En este punto se requiere de una argumentación razonada que justifique la elección del patrón de referencia en casos particulares (1).

Estas precauciones no invalidan el método, conducen sí a señalar que la identificación de las desigualdades de origen social en salud y el juicio que las 
califica de injustas es un proceso abierto a la constatación y a la interpretación. Se apoya en la medición de las desigualdades entre grupos, que es un complemento necesario a la de las desigualdades por individuos. Los postulados normativos sobre la injusticia que representan las inequidades en salud, requieren apoyarse en interpretaciones sobre la configuración social, es decir en análisis que desde posiciones de la ciencia social dan cuenta de las desigualdades sociales. Bajo la motivación valorativa, apoyada en explicaciones sobre las formas estructurales de la sociedad, se da paso a la búsqueda de una transformación de la sociedad. La evaluación de la equidad en salud se encuadra dentro de las prácticas tendientes a encontrar diferencias entre grupos y su explicación se centra en develar las inequidades sociales. Se convierte en una guía para la acción social colectiva.

Dentro de las categorías operativas que expresan posición social partimos en nuestra práctica en el Observatorio de Equidad en Salud de divisiones provisionales que deben ser comprobadas y mejoradas a medida que vayamos produciendo y analizando las variables disponibles. Utilizamos, dentro de la gama de las existentes, algunas que expresan condiciones de diferenciación con incidencia en las desigualdades en la salud y sus determinantes como el sexo, la edad y la posición socio-ocupacional, que pueden ubicarse dentro de lo que se entiende por "posición social”. Recurrimos a otras categorías que se refieren a diferencias en los ingresos, en el estándar de vida o al nivel socioeconómico como quintiles de ingreso, niveles educativos, estrato socioeconómico, niveles del índice Sisben y el índice de riqueza. El Sisben es el Sistema de Selección de Beneficiarios utilizado para la focalización de algunos programas sociales en Colombia. El índice de riqueza, utilizado internacionalmente en las Encuestas de Demografía y Salud (EDS), toma en cuenta los activos de los hogares y permite hacer una estratificación de los hogares. En la misma perspectiva se toma en cuenta la condición de pobreza y el desempleo que aunque tienen una variación coyuntural, pueden estar asociadas a las condiciones de salud y que tienen una incidencia en la relación entre grupo socioeconómicos y salud (7). Se incluyen también como variables diferenciadoras las referentes al lugar de residencia que están asociadas con condiciones socioeconómicas de las personas y los hogares (11) y la relativa a etnia, en tanto puede estar asociada con diferencias socioeconómicas. 
¿Qué evaluar en torno a los resultados en salud?

En la propuesta metodológica del Observatorio la evaluación de las inequidades en salud considera los siguientes temas:

1. Desigualdades en la morbilidad y discapacidad

2. Desigualdades en la mortalidad

3. Desigualdades en la autopercepción sobre el estado de salud.

Los indicadores de resultados en el estado de salud se refieren a la morbilidad, la mortalidad, la incapacidad y las diferencias en la percepción en del estado de salud, según grupos referidos a posiciones sociales. Incluyen los resultados agregados de las desiguales en salud, como expectativa de vida, morbilidad diferencial, mortalidad temprana, o mayor por ciertas patologías, asociadas a desventaja social (11).

Tenemos una enumeración de temas que no se corresponde con las nociones de salud, como expresión de un conjunto de capacidades o como es definida por el acta de constitución de la OMS: "La salud es un estado de completo bienestar físico, mental y social, y no solamente la ausencia de afecciones o enfermedades". El alcance de los indicadores propuestos está limitado por el marco de posibilidades que ofrece de la información disponible. Es deseable contar con mejor información para poder, por ejemplo, establecer las diferencias de la esperanza de vida por grupos socioeconómicos o calcular el índice propuesto por Gakidou, Murray y Frenk sobre las diferencias en la salud poblacional, no sólo globalmente sino para establecer diferencias entre estos grupos.

Parte de los mecanismos sociales que llevan a mantener las inequidades se encuentran en las prácticas de producción de información. Sobre los temas enunciados relativos a los temas de morbilidad y mortalidad carecemos de información sistemática y confiable para evaluar las inequidades. La información sobre morbilidad existente se ha convertido en Colombia en un monopolio de las entidades de aseguramiento, se ha convertido en privada y no puede tenerse acceso a ella. Llevamos más de 10 años sin información pública sobre el tema. Tan sólo se tiene información sobre morbilidad sentida obtenida de las encuestas de hogares.

En el caso de la mortalidad la información es más sistemática, confiable y continua. Ha habido avances importantes en su producción que ha permitido 
tener series sobre los últimos 10 años. Sin embargo, esta información no permite un análisis de diferenciales socioeconómicos en torno a clasificaciones importantes de posición social, a excepción del nivel educativo de las personas. La ausencia de información demográfica actualizada y confiable sobre volumen de población y su distribución por edad, sexo y características socioeconómicas ha impedido un análisis sobre las tasas diferenciales de muerte por estas variables. En el caso de los discapacitados, aunque ha habido igualmente avances su identificación se ha centrado en los "estratos” bajos lo que impide ver diferenciales sociales importantes.

Desigualdades injustas e inequidad en salud

Por último, quisiera hacer una breve referencia a algunas de las casillas de las figuras del modelo de análisis del Observatorio que no he mencionado y que llaman la atención sobre otros temas, relacionados con la equidad en salud, no incluidos en la consideración de las desigualdades entre individuos o grupos sociales.

La casilla que en el modelo se refiere al Contexto político e institucional señala la importancia de tomar en cuenta aspectos como el de la asignación presupuestal. Al respecto es ilustrativa la siguiente cita de Amartya Sen:

"La violación de la equidad en salud no se puede juzgar exclusivamente en función de la desigualdad en salud. En efecto, se puede argumentar que algunos de los problemas políticos más importantes de la promoción de la salud son profundamente dependientes de la asignación global de recursos a la salud, y no únicamente de acuerdos distributivos de la atención sanitaria (por ejemplo, el "racionamiento" de la atención sanitaria y de otros determinantes de la salud), en los cuales parece estar concentrada en la actualidad gran parte de la literatura sobre la equidad en salud. Los recursos son fungibles y los acuerdos sociales pueden facilitar la salud de los pobres, a costa no solo de la atención sanitaria o de los logros de salud de otras personas, sino también a través de un acuerdo social diferente o de una modificación de la asignación de recursos. La magnitud de la desigualdad en salud no puede proporcionarnos información suficiente para evaluar la equidad en salud". (5).

También la inclusión de esta casilla convoca a tomar en cuenta las condiciones de la participación social para adoptar las decisiones en torno a la salud, el tema de la democracia para decidir las políticas de salud no puede quedar por fuera de la evaluación de la equidad en salud.

Otro asunto igualmente necesario en la evaluación de la equidad es el de la eficiencia social de los servicios de salud, que está incorporado al tema que destaca la casilla de la derecha de las figuras 1 y 2: Consecuencias sociales 
y económicas de la inequidad en salud. La consideración interrelacionada de los indicadores de respuesta (insumos y procesos) y del estado de salud (resultados) da lugar al análisis complementario de la eficiencia en los logros sociales diferenciales en la salud de la población. Bajo la perspectiva de la equidad el tema de la eficiencia tiene también un papel activo en la medida en que la adecuada utilización de los recursos escasos favorece el mejoramiento de las condiciones de salud de los más desfavorecidos

\section{REFERENCIAS}

1. Fresneda O. Evaluar las inequidades en salud: propuesta de indicadores para el Observatorio de Equidad en Calidad de Vida y Salud para Bogotá. Restrepo D.,ed. Equidad y Salud, debates para la acción, Bogotá, CID, Universidad Nacional de Colombia, Secretaría Distrital de Salud; 2007: 103-143.

2. Diderichsen F, Hallqvist J. Social inequalities in health: some methodological considerations for the study of social position and social context. Arve-Parès B, ed. Inequality Health - A Swedish Perspective, Stockholm: Swedish Council for Social Research; 1998: 25-39.

3. Diderichsen F., Evans T., Whitehead M. Bases sociales de las disparidades en salud. F, Evans T, Whitehead M, Diderichsen F, Bhuiya A, Wirth M, ed. Desafío a la falta de equidad en salud: de la ética a la acción, Washington, Publicado por la Organización Panamericana de la Salud y la Fundación Rockefeller, Publicación científica y técnica No. 585; 2002: 13-25.

4. GEGA (Global Equity Gauge Alliance-Alianza Global para el Monitoreo de la Equidad). El monitoreo de la Equidad, conceptos, principios y pautas. Publicado por la Alianza Global para el Monitoreo de la Equidad y Consorcio de Sistemas de Salud, Durban, Sudáfrica; 2003.

5. Sen A. ¿Por qué la equidad en salud? Revista Panamericana de Salud Pública; 2002;11:302-309.

6. Whitehead M. Equity and Ethics in Health, World Health Organization. Regional Office for Europe. Copenhague; 1994: 1-17.

7. Evans T, Margaret W, Diderichsen F, Bhuiya A, Wirth M, ed. Desafío a la falta de equidad en salud: de la ética a la acción, Washington, DC.: Fundación Rockefeller; Organización Panamericana de la Salud; 2002. (Publicación Científica y Técnica No. 585).

8. Gakidou EE, Murray CJ, Frenk J. Defining and measuring health inequality: an approach based on the distribution of health expectancy. Bull World Health Organ 2000;78(1):42-54.

9. Braveman P, Gruskin S. Defining equity in health. J Epidemiol Community Health 2003;57(4):254-8.

10. Murray CJ, Gakidou EE, Frenk J. Health inequalities and social group differences: what should we measure? Bull World Health Organ 1999;77(7):537-43.

11. Martínez F. Enfoque para el análisis y la investigación sobre equidad-inequidad en salud. Revisión conceptual. Restrepo D.,ed. Equidad y Salud, debates para la acción, Bogotá, CID, Universidad Nacional de Colombia, Secretaría Distrital de Salud; 2007. p. 69-101. 